\title{
AVALIAÇÃO DA FUNÇÃO VESTIBULAR NA VERTIGEM PAROXÍSTICA BENIGNA DA INFÂNCIA
}

\author{
Adriana Marques da SILVA ${ }^{1}$ \\ Suelen CESARONI ${ }^{2}$ \\ Mauricio Malavasi GANANÇA ${ }^{3}$ \\ Heloisa Helena CAOVILLA ${ }^{4}$
}

${ }^{1}$ Fonoaudióloga Doutoranda em Distúrbios da Comunicação Humana pela Universidade Federal de São PauloUNIFESP. adriana10marques@gmail.com

${ }^{2}$ Fonoaudióloga do Setor de Otoneurologia no Instituto Penido Burnier - Campinas - SP. suelen.cesaroni@gmail.com

${ }^{3}$ Professor Titular de Otorrinolaringologia da Universidade Federal de São Paulo - UNIFESP - Departamento de Otorrinolaringologia e Cirurgia de Cabeça e Pescoço. mauricio.gananca@globo.com

${ }^{4}$ Professora Livre Docente da Disciplina de Otoneurologia da Universidade Federal de São Paulo - UNIFESP. heloisa.caovilla@globo.com

Trabalho realizado no Curso de Fonoaudiologia da Universidade Federal de São Paulo - UNIFESP - São Paulo (SP) Brasil.

Endereço para correspondência

Adriana Marques da Silva

R. Pedro de Toledo, 947, Vila Clementino, São Paulo (SP), Brasil, CEP: 04039-002.

E-mail: adriana10marques@gmail.com

Recebido em: 20/11/2015 - Aprovado em: 14/07/2016 - Disponibilizado em: 18/12/2016

\section{RESUMO}

Objetivo: Verificar a utilidade da eletronistagmografia computadorizada e da prova de auto-rotação cefálica na identificação de sinais e sintomas de disfunção vestibular em crianças com hipótese diagnóstica de vertigem postural paroxística benigna infantil (VPBI). Métodos: Foram selecionadas 50 crianças, sendo 30 do gênero feminino e 20 do gênero masculino, com idades entre 5 a 12 anos, com hipótese diagnóstica de VPBI. O exame da função vestibular foi realizado por meio da eletronistagmografia computadorizada e da prova de auto-rotação cefálica. Resultados: Observaram-se sinais de disfunção vestibular nas crianças avaliadas. Observou-se alteração na prova de auto-rotação cefálica, prova calórica, sacadas fixas e randomizadas e presença de nistagmo espontâneo com olhos fechados. Todas as alterações foram diagnosticadas como de origem vestibular periférica. Conclusão: A eletronistagmografia computadorizada e a prova de auto-rotação cefálica são úteis para identificar sinais de disfunção vestibular em crianças com diagnóstico de VPBI.

Palavras-chave: Testes de função vestibular. Auto-rotação cefálica. Nistagmo. Vertigem. Crianças.

\section{VESTIBULAR FUNCTION EVALUATIONIN BENIGNPAROXYSMAL VERTIGOOF CHILDHOOD}

\begin{abstract}
Purpose: To verify the usefulness of computerized electronystagmography and active head rotation testing in the identification of signs and symptoms of vestibular dysfunction in children diagnosed with benign paroxysmal vertigo of childhood (BPVC). Methods: A total of 50 children diagnosed with BPVC have been evaluated, 30 females and20 males, aged 5 to 12 years old. The vestibular function tests consisted of computerized
\end{abstract}


electronystagmography and active head rotation testing. Results: Signs of vestibular dysfunction have been found in the evaluated children. Active head rotation testing, caloric testing, fixed and randomized saccade test and presence of nystagmus with eyes close were the abnormalities found. All abnormalities were diagnosed as peripheral vestibular system impairments. Conclusion: Computerized electronystagmography and active head rotation testing are useful for the identification of vestibular dysfunction in children with BPVC.

Keywords: Vestibular function tests. Head rotation testing. Nystagmus. Vertigo. Children.

\section{INTRODUÇÃO}

$\begin{array}{lllr}\text { As desordens } & \text { vestibulares na } \\ \text { infância podem } & \text { interferir } & \text { no } \\ \text { desenvolvimento motor e de linguagem da } \\ \text { criança, acarretando } & \text { prejuízos } & \text { no } \\ \text { desenvolvimento da } & \text { postura, da } \\ \text { motricidade e no } & \text { desempenho } \\ \text { escolar(QUIRÓS, 1970; } & \text { AYRES, 1978; } \\ \text { GANANÇA, 1989; } & \text { GANANÇA; } \\ \text { CAOVILLA, 1989). }\end{array}$

Dentre as labirintopatias da infância está a vertigem paroxística benigna que ocorre aproximadamente em $50,0 \%$ das crianças com distúrbios vestibulares(GANANÇA; CAOVILLA, 1999).

A Vertigem Paroxística Benigna da Infância (VPBI) foi descrita pela primeira vez por Basser (1964) como surtos de vertigem súbita, que duram poucos minutos, acompanhada por palidez, sudorese, náusea e algumas vezes vômito; a criança não perde a consciência e não apresenta alterações auditivas (BASSER, 1964). Esta desordem vestibular foi associada com outros achados como dor de cabeça, antecedentes familiares de migrânea e classificada como uma desordem precursora do quadro de migrânea, visto que muitas crianças, em estudos a longo prazo, desenvolveram o quadro típico de migrânea na adolescência (BEDDOE, 1977;

UNERI;

\section{TURKDOGAN, 2003).}

O diagnóstico diferencial entre VPBI e outras afecções vestibulares, tais como enxaqueca basilar, epilepsia vestibular, neuronite e migrânea, é importante para a correta conduta médica no tratamento e prognóstico de cada caso (BATSON, 2004).

O diagnóstico da VPBI é geralmente realizado por meio da história clínica, exames audiológicos, vestibulares e radiológicos, quando necessário (GANANÇA; CAOVILLA, 1999); a inclusão da avaliação eletroencefalográfica à bateria de testes para o diagnóstico diferencial entre labirintopatias periféricas e centrais também pode ser útil (EVIATAR; EVIATAR, 1977).

Com o avanço tecnológico, a avaliação do exame vestibular ganhou o auxílio da análise computadorizada, que facilita o diagnóstico das alterações 
vestibulares e aumenta a sensibilidade da eletronistagmografia, analisando as respostas do paciente quanto aos parâmetros de fase, ganho e simetria(CAOVILLA et al., 1999). O teste de auto-rotação cefálica, de fácil realização e não invasivo, avalia o reflexo vestíbulo-ocular nas frequências naturais da movimentação da cabeça do dia-a-dia (CAOVILLA, 1996), e pode ser realizado com facilidade em crianças (ASSUNÇÃO; ALBERTINO; LIMA, 2002).

O objetivo de nossa investigação é verificar a utilidade da eletronistagmografia computadorizada e da prova de auto-rotação cefálica na identificação de sinais de disfunção vestibular em crianças com hipótese diagnóstica de Vertigem Paroxística Benigna.

\section{MÉTODO}

A presente pesquisa foi supervisionada pelo Setor de Equilibriometria da Disciplina de Otologia e Otoneurologia da Universidade Federal de São Paulo, consistindo em um estudo retrospectivo de exames vestibulares de pacientes, aprovado pela Comissão de Ética e Pesquisa da instituição, protocolo número 374/00.

Foram selecionadas 50 crianças, sendo 30 do gênero feminino e 20 do gênero masculino, com idades entre 5 e 12 anos, com hipótese diagnóstica de VPBI.

Todas as crianças foram
submetidas à anamnese, exame otorrinolaringológico e avaliação audiológica, antes de realizarem o exame da função vestibular.

Os pais dos pacientes foram orientados a submeterem seus filhos a uma dieta por 72 horas com abstinência de café, chá, chocolate e medicamentos não essenciais e a um jejum de 3 horas, antes da realização do exame vestibular. O exame vestibular foi executado por meio da eletronistagmografia computadorizada, utilizando procedimentos que incluem pesquisa do nistagmo de posicionamento, calibração dos movimentos oculares, nistagmo espontâneo com olhos abertos e fechados, nistagmo semi-espontâneo, nistagmo optocinético de direção fixa e alternada, movimentos sacádicos fixos e randomizados, rastreio pendular e prova calórica (CAOVILLA et al., 1999). Na prova de auto-rotação cefálica, a criança executou movimentos progressivamente mais rápidos com a cabeça, primeiramente no plano horizontal e depois no plano vertical, acompanhando os estímulos sonoros apresentados pelo computador, fixando um ponto luminoso à sua frente (CAOVILLA, 1996).

Os critérios de realização e interpretação do exame vestibular à 
nistagmografia computadorizada utilizou padrões definidos pela literatura (CAOVILLA et al., 1998)utilizando o equipamento Meta 4 Ultracomputerized ENG, da Micromedical Technologies, Inc., com o VORTEQ (Vestibular Ocular Reflex Equipament Test), para a prova de auto-rotação cefálica. O banco de dados do equipamento incluiu um padrão de normalidade para todos os testes vestíbulo-oculomotores realizados, analisando as respostas obtidas e definindo, automaticamente, as eventuais anormalidades funcionais encontradas.
Os achados foram detalhados, tabulados e apresentados por meio de frequência absoluta $(\mathrm{N})$ e relativa $(\%)$.

\section{RESULTADOS}

Os exames otorrinolaringológico e audiológico estiveram dentro dos padrões de normalidade para todas as crianças.

A Tabela 1 apresenta os achados do exame vestibular à eletronistagmografia computadorizada.

Tabela 1- Achados anormais à eletronistagmografia Computadorizada em 50 crianças diagnosticadas com Vertigem Paroxística Benigna da Infância.

\begin{tabular}{lll}
\hline Achados Anormais & N & \% \\
\hline Nistagmo espontâneo com olhos fechados & 1 & 2,0 \\
Aumento de latência das sacadas fixas & 6 & 12,0 \\
Aumento da latência das sacadas randomizadas & 6 & 12,0 \\
Auto-Rotação Cefálica & 5 & 10,0 \\
Aumento de Ganho do RVO horizontal & 5 & 10,0 \\
Aumento do ganho e atraso de fase do RVO horizontal & 7 & 14,0 \\
Aumento de ganho RVO vertical & 3 & 6,0 \\
Aumento de ganho do RVO horizontal e vertical & 7 & 14,0 \\
Aumento de ganho e atraso de fase do RVO horizontal e vertical & 0 & 0,0 \\
Prova Calórica & & \\
Hiper-reflexia bilateral & 1 & 2,0 \\
Hiporreflexia unilateral & 3 & 6,0 \\
Preponderância direcional do nistagmo & 1 & 2,0
\end{tabular}


Observou-se 39 crianças $(78,0 \%)$ com sinais de disfunção vestibular e 11 crianças $(22,0 \%)$ com resultados normais. As provas de nistagmo de posicionamento, nistagmo espontâneo com os olhos abertos, nistagmo semi-espontâneo, rastreio pendular e nistagmo optocinético de direção fixa e alternada não apresentaram alterações. A prova de autorotação cefálica apresentou resultados dentro dos padrões da normalidade em $22,0 \%$ das crianças e a prova calórica em $72,0 \%$

O topodiagnóstico nos 39 casos, que apresentaram alteração no exame vestibular, foi de vestibulopatia periférica, sendo que nenhum paciente apresentou sinais compatíveis com a hipótese diagnóstica de vestibulopatia central.

\section{DISCUSSÃO}

No estudo da função vestibular em crianças com Vertigem Paroxística Benigna, à eletronistagmografia computadorizada, observamos que $78,0 \%$ dos casos apresentaram sinais de disfunção vestibular, enquanto $22,0 \%$ apresentaram exame vestibular normal. $\mathrm{Na}$ literatura compilada, vários autores comentaram que o exame vestibular de crianças com VPBI pode estar alterado (BASSER, 1964; DUNN; SNYDER,1976; GANANÇA et al., 1998), mas outros autores revelaram que os testes vestibulares à eletronistagmografia não computadorizada têm, em geral, resultados normais (CYR; RUBIN, 1982; BOWER; COTTON, 1995).

A auto-rotação cefálica ativa é um teste rápido, de simples realização, não invasivo, que não causa desconforto ao paciente, pode ser realizado com facilidade em crianças e avalia o reflexo vestíbulo-ocular nas frequências fisiológicas de movimentação da cabeça, utilizadas na vida cotidiana (de 1 a $4 \mathrm{~Hz}$ ) (ASSUNÇÃO; ALBERTINO; LIMA, 2002).

Em nossa avaliação, a prova de auto-rotação cefálica identificou sinais de disfunção vestibular em $78,0 \%$ das crianças com VPBI, sendo $20,0 \%$ no reflexo vestíbulo-ocular horizontal, 20,0\% no reflexo vestíbulo-ocular vertical e $38,0 \%$ no reflexo vestíbulo-ocular vertical e horizontal. Autores descreveram aumento do ganho no reflexo vestíbuloocular horizontal e atraso da fase no reflexo vestíbulo-ocular vertical, em um paciente com VPPI (GANANÇA et al., 1998).

É frequente a ocorrência de pacientes com alteração na prova de autorotação cefálica e demais provas do exame vestibular normais (GANANÇA et al., 2009), devido ao fato de que esta prova mede o reflexo vestíbulo-ocular horizontal 
e vertical com estímulos de alta frequência, enquanto as outras provas do exame vestibular avaliam somente o reflexo vestíbulo-ocular horizontal a estímulos de baixa frequência (BLATT et al., 2008).

Autores afirmaram que a autorotação cefálica é uma prova importante no diagnóstico das disfunções vestibulares, aumentando a sensibilidade do exame(CAOVILLA, 1996; GANANÇA; CAOVILLA, 1996; GANANÇA; CAOVILLA; GANANÇA, 1997).

A prova calórica apresentou alterações em $28,0 \%$ das crianças por nós avaliadas: $20,0 \%$ de hiperreflexiabilateral, 6,0\% com hiporreflexia unilateral e 2,0\% com preponderância direcional do nistagmo.

Outros autores comentaram que a prova calórica frequentemente está comprometida e é a mais alterada, apresentando sinais do tipo hipo ou arreflexia vestibular, em crianças com VPBI à eletronistagmografia não computadorizada (BASSER, 1964; BUSIS, 1985; DUNN; SNYDER, 1976; KOENIGSBERGER et al., 1970; MEDINA et al., 1985; SOARES; AGUIRRE; FORMIGONI, 1994; KOSTIĆ et al., 2012). Por outro lado, autores que realizaram os testes vestibulares com eletronistagmografia computadorizada, encontraram a presença de hiper-reflexia à prova calórica (GANANÇA et al., 1998).

Encontramos alteração em 12,0\% dos casos à avaliação das sacadas fixas e em $12,0 \%$, das sacadas randomizadas.

É possível encontrar o nistagmo espontâneo de olhos abertos, como sinal de disfunção vestibular em crianças com VPBI, durante as crises vertiginosas, mas não durante os testes realizados (BUSIS, 1985). Em nosso estudo, 2,0\% das crianças apresentaram nistagmo espontâneo com os olhos fechados de pequena intensidade. Autores descreveram um paciente com VPBI que apresentou nistagmo espontâneo com os olhos fechados, muito intenso, com 19o/s à eletronistagmografia computadorizada (GANANÇA et al., 1998).

Quanto ao topodiagnóstico, observamos que todas as crianças com sinais de disfunção vestibular $(78,0 \%)$ foram diagnosticadas como portadoras de uma labirintopatia periférica. Alguns autores consultados discordam desses dados, descrevendo a possibilidade do encontro de sinais centrais em pacientes com VPBI (MIRA et al., 1984; CORREA, 1980).

A partir dos dados obtidos, pudemos verificar que a maioria das crianças com VPBI apresentaram sinais de disfunção vestibular, sendo que as provas de autorotação cefálica e prova calórica à 
eletronistagmografia computadorizada foram as mais alteradas.

\section{CONCLUSÃO}

A eletronistagomografia computadorizada e a prova calórica de autorotação cefálica são úteis para identificar sinais de disfunção vestibular em crianças com diagnóstico de Vertigem Paroxística Benigna da Infância.

\section{REFERÊNCIAS}

ASSUNÇÃO, A.R.M.; ALBERTINO, S.; LIMA, M.A.A.T.Auto-rotação cefálica ativa em pacientes com tontura. Bras

Otorrinolaringol. 2002;68:(1):57-63. AYRES,A.J.Learning disabilities and the vestibular system. Learn Disabil. 1978;11:30-41.

BASSER, I.S.Benign paroxysmal vertigo of childhood. Brain. 1964;141-52.

BATSON, G.Benign paroxysmal vertigo of childhood: a review of the literature.

PaediatrChild Health. 2004;9(1):31-4.

BEDDOE, G.M. Vertigo in childhood. OtolaryngolClin N Am. 1977;10(1):13944.

BLATT, P.J.; SCHUBERT, M.C.; ROACH, K.E.; TUSA, R.J. The reliability of the vestibular autorotation test (VAT) in patients with dizziness. J Neurol Phys Ther. 2008;32(2):70-9.

BOWER, C.M.; COTTON, R.T.The spectrum of vertigo in children. Arch Otolaryngol Head Neck Surg. 1995;121:911-15.
BUSIS, S.N. Dizziness in children. Eng Report. 1985.

CAOVILLA, H.H.Da rotação cefálica ativa em pacientes vertiginosos sem sinais de disfunção vestibular à vectoeletronistagmografia. [Tese de LivreDocência em Otoneurologia]. São Paulo: UNIFESP-EPM; 1996.

CAOVILLA, H.H.; GANANÇA, M.M.; MUNHOZ, M.S.L.; SILVA, M.L.G. Equilibriometria clínica. São Paulo: Atheneu; 1999. 158p.

CORREA,

A.Transtornosdelequilibrioenlosninos. Rev Child Pediatr. 1980;51(1): 27-9.

CYR, D.G.; RUBIN, A.The evaluation of vestibular function in infants and young children. Insights in Otolaryngology. 1982;7(2):1-8.

DUNN, D.W.; SNYDER, H.Benign paroxysmal vertigo of childhood. Am J Dis Child.1976;130:1099-100.

EVIATAR, L.; EVIATAR, A. Vertigo in children: differential diagnosis and treatment. Pediatr. 1977;59(6):833-8.

GANANÇA, F.F.; GANANÇA, C.F.; CAOVILLA; H.H.; GANANÇA, M.M.; ALBERNAZ, P.L.Active head rotation in benign positional paroxysmal vertigo. Braz J Otorhinolaryngol. 2009;75(4):586-92.

GANANÇA, M.M.Da vestibulometria em crianças com distúrbios de linguagem[Tese de Doutorado]. São Paulo, Escola Paulista de Medicina, 1989.

GANANÇA, M.M., CAOVILLA, H.H. Labirintopatias infantis. RevBrasClin Terap. 1989;18:350-69.

GANANÇA, M.M.; CAOVILLA, H.H. Labirintopatias na infância. In: Caldas N, Caldas Neto S, Sih T.Otologia e Audiologia em Pediatria. Revinter: Rio de Janeiro; 1999. p. 277-86. 
GANANÇA, M.M.; CAOVILLA;

H.H.Vestibulopatias: sensibilidade diagnóstica dos métodos de avaliação. Rev

Bras Med Otorrinolaringol.

1996;3(2):128.

GANANÇA, M.M.; CAOVILA, H.H.;

GANANÇA, F.F. Active head rotation

(VORTEQ) in dizzy patients with or

without ENG abnormalities. In:

IBEROAMERICAN CONGRESS OF

OTONEUROLOGY, Cancún, México, 1997. Anals. p.73.

GANANÇA, M.M.; CAOVILLA, H.H.; MUNHOZ, M.S.L.; SILVA, M.L.G.;

FRAZZA, M.M.; GANANÇA, F.F.;

GANANÇA, C.F. Xeque-mate nastonturas

IV. Vertigem paroxística benigna infantil

em xeque.ActaAwho. 1998;17(3):139-49.

KOEHLER, B.

Benignparoxysmalvertigoofchildhood: a migraineequivalent. Eur J Pediatr.

1980;134:149-51.

KOENIGSBERGER, M.R.;CHUTORIAN, A.M.; GOLD, A.P.; SCHVEY, M.S. Benign paroxysmal vertigo of childhood.

Neurology. 1970;20:1108-13.

KOSTIĆ,M.;TROTIĆ,R.;JANKES, K.R.;LEVENTIĆ,M. Benign paroxysmal vertigo in childhood. CollAntropol.

2012;36(3):1033-6.

KRAMS, B.; ECHENNE, B.; LEYDET, J.; RIVIER, F.; ROUBERTUIE, A. Benign paroxysmal vertigo of childhood: long-term outcome. Cephalalgia. 2010;31(4):439-43.

MEDINA, M.C.; PÈREZ, A.V.; MARTIN, V.L.;CASTROVIEJO,I.P.Vértigo paroxístico benigno de lainfancia: estudo de 36 casos. Arch DominPediatr. 1985;21(2): $57-9$.

MIRA, E.; PIACENTINO, G.; LANZI, G.; BALOTTIN, U.; FAZZI, E. Benign paroxysmal vertigo in childhood: a migraine equivalent. ORL.1984;46:97-104.
QUIRÓS, J.B. hablaLasllamadas afasias infantiles y elniño que no: sus rel aciones conlassorderascentrales.Fonoaudiológica. 1970;16(1):83-8.

SOARES, I.P.; AGUIRRE, B.R.; FORMIGONI, L.G.Vertigem na infância.Rev Bras Med Otorrinolaringol. 1994;60 (2):92-7.

UNERI, A.; TURKDOGAN, D.Evaluation of vestibular functions in children with vertigo attacks.Arch Dis Child. 2003;88:510-1. 